\title{
Development and regulation of exosome-based therapy products
}

\author{
Elena V. Batrakova* and Myung Soo Kim
}

\begin{abstract}
Recently, various innovative therapies involving the ex vivo manipulation and subsequent reintroduction of exosome-based therapeutics into humans have been developed and validated, although no exosome-based therapeutics have yet to be brought into the clinic. Exosomes are nanosized vesicles secreted by many cells that utilize them for cell-to-cell communications to facilitate transport of proteins and genetic material. Comprised of cellular membranes with multiple adhesive proteins on their surface, exosomes offer distinct advantages that exceptionally position them as highly effective drug carriers. Additionally, exosomes can exert unique biological activity reflective of their origin that may be used for therapy of various diseases. In fact, exosomes have benefits of both synthetic nanocarriers and cell-mediated drug delivery systems, and avoid their limitations. This concise review highlights the recent developments in exosomebased drug delivery systems and the main regulatory considerations for using this type of therapeutic in clinic. () 2016 Wiley Periodicals, Inc.
\end{abstract}

How to cite this article:

WIREs Nanomed Nanobiotechnol 2016, 8:744-757. doi: 10.1002/wnan.1395

\section{INTRODUCTION}

A $\mathrm{n}$ increasing amount of nanomaterials are being designed to aid in the transport of diagnostic and therapeutic agents through biologic barriers. A common approach for building a drug delivery system is to incorporate the drug within the nanocarrier that results in increased solubility, metabolic stability, and improved circulation time. Several formulations are being studied in clinical trials, or have already been approved by the FDA for use in humans. ${ }^{1,2}$ However, conventional nanoparticles normally are cleared rapidly from the circulation by the mononuclear phagocyte system (MPS). ${ }^{3}$ Extending the circulation time of these carriers can be accomplished by decorating these carriers with water-soluble polymers such as polyethylene glycol (PEG). Although PEGylation decreases clearance by the MPS, it also reduces

\footnotetext{
*Correspondence to: batrakov@email.unc.edu
}

Center for Nanotechnology in Drug Delivery, Eshelman School of Pharmacy, University of North Carolina at Chapel Hill, Chapel Hill, NC, USA

Conflict of interest: The authors have declared no conflicts of interest for this article. interaction of the nanoformulation with target and barrier cells, thus decreasing the drug biodistribution in disease tissues. ${ }^{4-6}$ In addition, development of an immune response to the PEG corona significantly increases the clearance of PEGylated drug nanocarriers. ${ }^{7-9}$ In extreme cases, severe allergic reactions to PEG due to immediate exposure to reactive antibodies in the blood were reported. ${ }^{10}$ With this foundation, exosomes have recently emerged as a promising drug delivery system for various smalland high-molecular-weight therapeutic agents with low immunogenicity, low toxicity, and high therapeutic efficacy. ${ }^{11}$ It was suggested that collected from patients' tissues or blood, allogenic exosomes should have immune-privileged status with low clearance by MPS. Furthermore, the cell source of these extracellular vesicles (EVs) may determine their biodistribution, indicating that they may possess an innate homing ability. In addition, exosomes can be targeted to specific disease tissues via modifications of their parental cell, or incorporating of a tissue-specific vector moiety vector into exosomes. Consequently, an increasing number of researchers investigate and utilize exosomes for the delivery of low-molecularweight therapeutics, nucleic acids, proteins, and even 
viruses. The overall scheme of different approaches to using exosome-based therapeutic formulations is depicted in Figure 1.

\section{BIOGENESIS OF EXOSOMES}

The unique properties of exosomes can be attributed to their biogenesis; the classical view of exosome biogenesis holds that they are accumulated initially in multivesicular sacs, i.e., multivesicular bodies (MVBs) followed by fusion with the plasma membrane and their release. ${ }^{11}$ Exosomes' close relative, microvesicles, are believed to bud directly from the plasma membrane. They are greater in size $(100-500 \mathrm{~nm})$ than exosomes $(40-100 \mathrm{~nm})$ and have slightly different protein and lipid content. ${ }^{12}$ Consequently, exosomes and microvesicles are currently believed to have endosomal and plasma membrane origin, respectively. Larger vesicles (500-1000 nm) are considered to be apoptotic bodies. Many investigations, especially in the field of drug delivery, utilize both exosomes and microvesicles, defining them as extracellular vesicles, because a complete separation and purification of each type of vesicles is extremely laborious and difficult, if not impossible. ${ }^{13}$

Many cell types actively secrete exosomes, in particular, cells of the immune system such as dendritic cells (DCs), ${ }^{14}$ macrophages, ${ }^{15} \mathrm{~B}$ cells, ${ }^{16}$ and T cells, ${ }^{17}$ as well as mesenchymal stem cells (MSCs), ${ }^{18}$ endothelial cells, ${ }^{19}$ and epithelial cells. ${ }^{20}$ Exosomes and other types of microvesicles are also secreted by a variety of cancer cells. ${ }^{21}$ Exosomes can be isolated from conditioned cell culture media or bodily fluids by differential centrifugation, ${ }^{14}$ filtration paired with centrifugation, ${ }^{14}$ immunoaffinity, ${ }^{14}$ size exclusion chromatography, ${ }^{22}$ or polymer-based precipitation, ${ }^{23,24}$ as well as microfluidic technologies $^{25,26}$ utilizing principles from the aforementioned methods. Each method has its advantages and disadvantages, requires different methods of preprocessing of samples, and produces exosome preparations of varying purity and quality. ${ }^{24,27,28}$ The user may choose a method for exosome isolation based on the intended downstream use. Interestingly, recently a novel approach designed to isolate exosomes from freshly removed and frozen mouse and human brain tissues secreted into brain extracellular space was developed. ${ }^{29}$ These exosomes were isolated from brain tissue treated with papain by subsequent centrifugation.

\section{EXOSOME CHARACTERIZATION}

Exosomes can be characterized by the size and protein and lipid content. Different techniques were developed for the characterization of exosomes. Among them are flow cytometry, Western blotting, nanoparticle tracking analysis, dynamic light scattering (DLS), mass spectrometry (MS), and several microscopy techniques. ${ }^{30}$ The International Society for Extracellular Vesicles (ISEV) published a position paper in 2014, in which the characterization of exosomes is recommended by the presence of exosomeassociated surface markers as well as the absence of proteins not associated with exosomes. ${ }^{31}$ Exosomal surface markers include TSG101, Alix, flotillin 1, tetraspanins (CD9, CD63, CD81), integrins, and cell adhesion molecules. ${ }^{31}$ Regarding lipid content, exosomes are highly enriched in cholesterol, sphingomyelin, and hexosylceramides at the expense of phosphatidylcholine and phosphatidylethanolamine. ${ }^{32}$ The fatty acids in exosomes are mostly saturated or monounsaturated. Together with the high concentration of cholesterol, this may account for lateral segregation of these lipids into exosomes during their formation at MVBs.

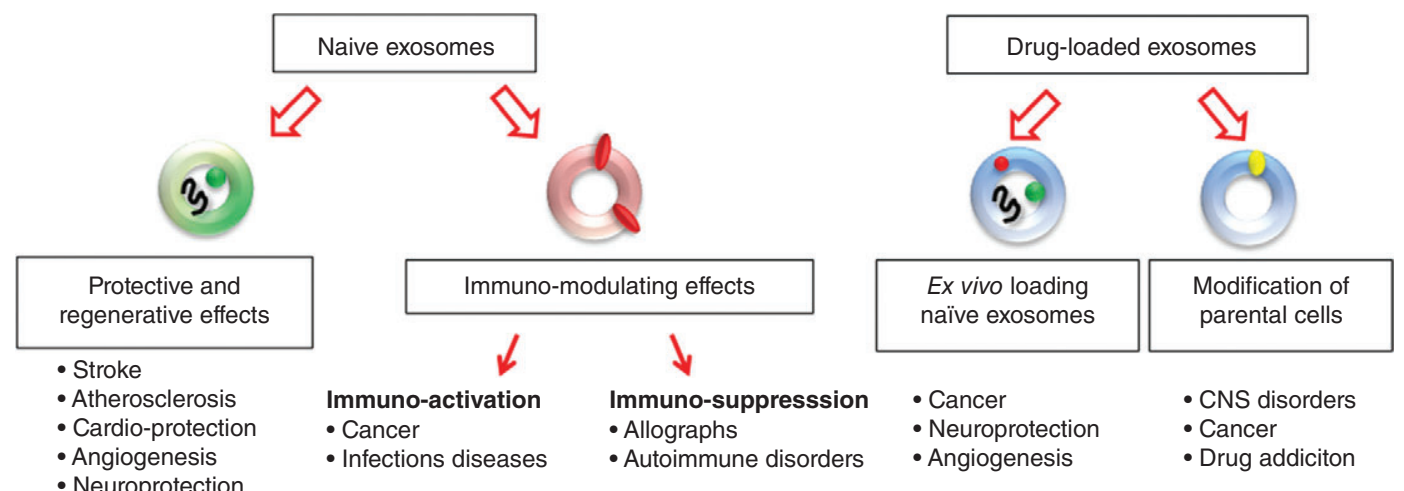

FIG URE 1 | Overview of approach to using exosome-based therapeutic formulations. 


\section{PROTECTIVE AND REGENERATIVE EFFECTS OF EXOSOMES}

Exosomes represent important vehicles of intercellular communication in between cells, and play a vital role in regulating a broad range of physiological and pathological cellular processes. ${ }^{33}$ Some of these functions may be utilized for therapeutic purposes.

One of the major candidates for this role is exosomes derived from MSCs. ${ }^{34-42}$ These cells may originate from bone marrow, adipose tissue, cord blood, and other origins. Thus, MSCs-derived exosomes were reported to produce significant cardioprotective paracrine effects against myocardial ischemia/reperfusion injury in pig and mouse models. ${ }^{35,36}$ These exosomes were also beneficial in pulmonary hypertension (HP). HP is a kind of malignant pulmonary vascular disease characterized by an increase in pulmonary artery pressure, which may lead to heart failure and even death. Interestingly, MSCs-derived exosomes directly suppressed early pulmonary inflammation and vascular remodeling ${ }^{37}$ through the suppression of hyperproliferative pathways, including signal transducer and activator of transcription 3 (STAT3)-mediated signaling. Furthermore, it was reported that stem cells-derived EVs have the ability to induce angiogenic programs in quiescent endothelial cells. ${ }^{43}$ Furthermore, MSCs-derived exosomes were also shown to have neuroprotective effects in stroke. It was demonstrated that stroke induces changes in the microRNA (miRNA) profile of these exosomes, ${ }^{4,45}$ especially in miRNAs that actively participate in the recovery process after stroke. ${ }^{46}$ MSCs-derived exosomes transferred their therapeutic factors to recipient cells, altered gene expression, and thereby promoted neurite growth in rat primary neurons. ${ }^{39}$ Next, a hepatic regeneration was shown by use of MSC-derived exosomes in drug-induced liver injury models. ${ }^{47,48}$ The higher survival rate was associated with upregulation of the priming-phase genes during liver regeneration, which subsequently led to higher expression of proliferation proteins (PCNA and cyclin D1) in the exosome-treated group. Therapeutic effects of exosomes derived from human adipose tissue-derived MSCs were also reported for the treatment of Alzheimer's disease (AD). ${ }^{38}$ It was demonstrated that these exosomes carry enzymatically active neprilysin (NEP), the most important enzyme that degrades $\beta$-amyloid $(\mathrm{A} \beta)$ peptide plugs in the brain. MSCs-derived exosomes decreased both intracellular and extracellular $\mathrm{A} \beta$ levels in a neuroblastoma cell line $\mathrm{N} 2 \mathrm{~A}$ in vitro.

Along with stem cells-derived exosomes, protective and regenerative effects were reported for exosomes released by endothelial cells. ${ }^{49}$ Atherosclerosis, the underlying cause of myocardial infarction (MI) and stroke, occurs predominantly in predisposed spots in the large arteries. Exosomes derived from endothelial cells were suggested to be a promising strategy to combat atherosclerosis. ${ }^{49}$ Systemic administration of exosomes released from human umbilical vein endothelial cells (HUVECs) reduced atherosclerotic lesions in mice fed a high-fat diet. Exosomes secreted by shear-stress-stimulated HUVECs were enriched in multiple miRNAs. The delivery of these miRNAs to smooth muscle cells (SMCs) resulted in controlled target gene expression and reduction of atherosclerotic lesion formation in the mouse aorta. ${ }^{49}$ Next, exosomes secreted from cardiosphere-derived cells (CDCs) were also shown to produce a range of diverse cardioprotective effects, including anti-inflammatory, antioxidative, antiapoptotic, antifibrotic, and cardiomyogenic effects. ${ }^{42,50,51}$ CDCs-released exosomes stimulated angiogenesis, promoted cardiomyocyte proliferation, and decreased programmed cell death in vitro. The regenerative capacity of these exosomes was demonstrated in a model of chronic MI in rats. ${ }^{52}$ These diverse effects were attributed to the ability of exosomes to enhance neovascularization, cardiomyocyte survival, and to reduce fibrosis after occurrence of cardiac infarction via paracrine mechanisms. ${ }^{53}$

Finally, MVBs have also been identified in plants, and leaderless secreted proteins can be released in vesicles, as described recently. ${ }^{54,55} \mathrm{Ju}$ et al. showed protective effects of exosome-like nanoparticles isolated from crushed grapes. ${ }^{56}$ In particular, the oral administration of exosome-like nanoparticles from grapes to mice led to the significant proliferation of the intestinal epithelium. These exosomes are being tested for their effects on oral mucositis and related pain after radiotherapeutic and chemotherapeutic treatment of head and neck cancers in an ongoing clinical trial (NCT01668849).

\section{IMMUNE-MODULATING PROPERTIES OF EXOSOMES}

Immunotherapy against various cancer tumors with autologous exosomes is the area of the most developed exosome-based therapeutics. ${ }^{57}$ Thus, two Phase I clinical trials using exosomes for the treatment of non-small-cell lung (NSCL) and melanoma cancers have been successfully completed. ${ }^{58,59}$ Usually, exosomes released by activated antigen-presenting cells, such as DCs, macrophages, $\mathrm{T}$ lymphocytes, or B cells, are utilized. The presence of MHC class I and 
II, as well as T-cell co-stimulatory molecules on the surface of these exosomes is an important mechanism of antigen presentation. ${ }^{60}$ In addition, immune response cells that were exposed to cancer cells were shown to package cellular components from these cancer cells into exosomes and trigger an antitumor response by presenting tumor antigens to immune cells. ${ }^{61-67}$

In particular, DCs-secreted exosomes incubated with human breast adenocarcinoma cells (SK-BR-3) were demonstrated to induce tumor-sensitized $\mathrm{T}$ cells to secrete high levels of Interferon- $\gamma(\mathrm{IFN}-\gamma){ }^{62}$ Recently, Di Bonito et al. ${ }^{68}$ reported that HEK293T cells, which were transfected with an antigen, HPVE7, produced exosomes loaded with the antigen. These HPV-E7-loaded exosomes produced a similar cytotoxic CD8+ T-cell response in C57BL/6 mice as compared to virus-like particles containing the same antigen when systematically administered in drugequivalent amounts. Next, Qazi et al. ${ }^{63}$ observed a significant anticancer activity of exosomes secreted by DCs that were exposed to chicken egg ovalbumin (OVA). These exosomes elicited specific transgenic Tcell proliferation in vitro. Furthermore, T-cellreleased exosomes were shown to destroy tumor stroma and prevent tumor invasion and metastases. ${ }^{64}$ In addition to the improved immune responses, Tcell-derived EVs were demonstrated to modulate endothelial cell responses to vascular endothelial growth factor (VEGF) and alter tube formation and gene expression in target endothelial cells.

Importantly, the induction of immune responses may be mediated not only by the bioactive lipids and proteins present in exosomes but also by exosomeand EV-associated RNAs. ${ }^{65}$ Contained inside exosomes, small noncoding RNAs (miRNAs) play a key role in mediating biological functions owing to their prominent role in gene regulation. Thus, Aucher et al. ${ }^{66}$ reported that human macrophages can transfer miRNAs to hepatocarcinoma cells (HCCs) and functionally inhibit proliferation of these cancerous cells. The transport of these miRNA was associated with EVs.

Aside of antitumor effects, DCs-derived exosomes were also shown to improve immune responses to difteria and Leishmania infections. ${ }^{69,70}$ Furthermore, exosomes that were found in human breast milk can boost the immune response and alter the T-cell balance toward a regulatory phenotype. ${ }^{71,72}$

Finally, exosomes can be used for immunosuppressive purposes. Thus, immature DCs express a low ratio of co-stimulatory molecules to $\mathrm{co}^{-}$ regulatory molecules on their surface, and are therefore immunosuppressive. A treatment with exosomes derived from these in vitro-generated immunosuppressive DCs reversed early-onset collageninduced arthritis even more effectively than the treatment with parental cells. ${ }^{73}$ Immunosuppressive exosomes have also been used in transplantation to inhibit the alloantigen-related immune responses such as allograft rejection. Thus, the donor-specific allograft immunosuppression was induced by the cotreatment with donor DCs-derived exosomes and a drug that blocks the maturation of recipient DCs. ${ }^{74}$ DCs-derived exosomes injected before transplantation significantly prolong heart allograft survival in congenic and fully MHC-mismatched Lewis rats. In addition, cardiac endothelial cell-derived exosomes were shown to induce specific regulatory B cells with immune suppressor functions. ${ }^{19}$ Overall, exosomes are potent immune regulators and may be utilized for the design of vaccine adjuvants and therapeutic intervention strategies to modulate immune responses.

\section{THERAPEUTIC EFFECTS OF DRUG- LOADED EXOSOMES}

As exosomal carriers can provide advantages of both cell-based drug delivery and nanotechnology, interest in using exosomes for drug delivery has exploded in recent years. ${ }^{75-77}$ Similar to viruses, these remarkable carriers are capable of traveling from one cell to another, easily passing their contents across the cell membrane owing to their unique characteristics, and delivering their cargo in a biologically active form. Noteworthy, exosomes possess an inherent ability to cross biological barriers, including the most difficult to penetrate, the blood-brain barrier (BBB).

Exosomes have been exploited as drug delivery vehicles for small-molecule therapeutic agents in several investigations. ${ }^{78-85}$ In one of the first reports, exosomes loaded with an anti-inflammatory compound, curcumin, were shown to protect mice from lipopolysaccharides-induced brain inflammation. ${ }^{78,79}$ The incorporation of curcumin in exosomes improved its solubility, increased circulation time, preserved drug therapeutic activity, and enhanced drug transport across the BBB. In another study, exosomes or exosome-like vesicles loaded with different chemotherapeutics, doxorubicin (Dox) or paclitaxel (PTX), were shown to traffic to tumor tissues and reduce tumor growth in mice without adverse effects observed with the equipotent free drug. ${ }^{80-82}$ Notably, the therapeutic effects of Dox-loaded exosomemimetic nanovesicles were greater than the commercially available Dox-loaded liposomes, Doxil; the 
liposomal formulation was inefficient in reducing tumor growth in this model. ${ }^{80}$ Pascucci et al. observed that PTX-treated MSCs mediated strong antitumorigenic effects because of their capacity to take up the drug and later release it in EVs. ${ }^{84}$ In this study, PTX-loaded EVs induced a dosedependent inhibition of human pancreatic adenocarcinoma cell (CFPAC-1) proliferation and 50\% inhibition of tumor growth in vivo. Furthermore, PTXloaded exosomes released from macrophages were developed for PTX to treat multidrug resistant cancers. ${ }^{86}$ Exosomes were loaded with PTX using three different methods: incubation at room temperature, electroporation, and mild sonication. It was demonstrated that a reformation of the exosomal membranes upon mild sonication to preserve structure and content of exosomal membranes resulted in high loading efficiency and sustained drug release. Importantly, the incorporation of PTX into exosomes (exoPTX) resulted in more than 50 times increases in cytotoxicity in drug-resistant MDCKMDR1 (Pgp+) cells. The increase in PTX cytotoxicity afforded by exoPTX was greater in Pgp-overexpressing cells than in their sensitive counterparts MDCK $_{\mathrm{wt}}$ cells. ${ }^{86}$ Strikingly, the obtained results showed a nearly complete co-localization of airway-delivered exosomes with cancer cells in a model of murine Lewis lung carcinoma (LLC) pulmonary metastases. Consequently, exoPTX demonstrated superior inhibition of pulmonary metastases growth in LLC mouse model. It was hypothesized that three mechanisms are likely to have significant impact on exoPTX anticancer activity, i.e., preferential accumulation of exoPTX in cancer cells, efficient delivery of the incorporated cargo into target cancer cells, and bypassing Pgp-mediated drug efflux in resistant cancer cells. In another investigation, exosomes derived from brain endothelial cell line, bEND.3, were loaded with anticancer drugs (Dox and PTX) and used for systemic delivery across the $\mathrm{BBB}$ to treat gliomas in a xenotransplanted zebrafish model of brain cancer. ${ }^{83}$ Exosome-delivered anticancer agents significantly decreased the fluorescent intensity of the cancer cells and tumor growth marker. Finally, membrane vesicles loaded with hydrophobic photosensitizers exhibited superior phototherapeutic effects compared to the polymerbased synthetic nanoparticles. ${ }^{85}$ Specifically, these membrane vesicles were able to fuse with the plasma membrane of cancer cells more effectively than polymer-based synthetic nanoparticles. Drug-loaded membrane vesicles accomplished co-delivery of hydrophobic and hydrophilic compounds into the cellular membrane and cytosol, respectively, largely bypassing the endosome/lysosome pathway and thereby enhancing the drug therapeutic efficacy in both spheroids and in vivo tumors. ${ }^{85}$

Another therapeutic avenue involves the use of exosomes to deliver exogenous small interfering RNA (siRNA). ${ }^{37,87-94}$ Exosomes were suggested as carriers for nucleic acids transfer, as they known to naturally deliver mRNA, miRNA, various noncoding RNA, mitochondrial DNA, and genomic DNA. ${ }^{95,96}$ Similar to the incorporation of genetic material into living cells, electroporation of purified exosomes was proposed for loading of exogenous RNA. ${ }^{91-94,97}$ Alvarez-Erviti et al. pioneered this method using electroporation DC-derived exosomes in the presence of siRNAs. ${ }^{94}$ The same method was used to load exosomes with miRNA to epidermal growth factor receptor (EGFR) that is expressed breast cancer cells. ${ }^{98}$ Next, exosomes are known to carry a negative surface charge, hence precluding electrostatic siRNA complexation. Precomplexation of siRNA via cationic liposomes followed by fusion with isolated exosomes has been suggested for their loading with siRNA by Wahlgren et al. ${ }^{87}$ The group reported the efficient silencing of the target MAPK gene in monocytes and lymphocytes using peripheral blood-borne exosomes with incorporated exogenous siRNAs. ${ }^{87} \mathrm{In}$ another investigation, Shtam et al. introduced two different exogenous siRNAs against RAD51 and RAD52 into exosomes derived from HeLa cells. ${ }^{88}$ The exosome-delivered siRNA against RAD51 was functional and caused massive cell death of recipient cancer cells. The effect of EVs loaded with siRNA targeted to miR-150, an oncomir, due to its promotional effect on VEGF has also been reported. ${ }^{89,90}$ It was demonstrated that the neutralization of miR-150 downregulated VEGF levels in mice and attenuated angiogenesis. O'Brien et al. ${ }^{99}$ engineered EVs loaded with miR-134, which is heavily downregulated in breast cancer tissue as compared to healthy tissue. It was demonstrated that miR-134-loaded EVs were able to reduce STAT5B and Hsp90 levels in target breast cancer cells and reduce cellular migration and invasion. Furthermore, miR-134-loaded EVs enhanced sensitivity of these cancer cells to the antiHsp90 drugs, 17-AAG and PU-H71. Noteworthily, this treatment did not significantly affect the cell proliferation or cisplatin-induced apoptosis, unlike the direct transfection of the target cells with miR-134. Interestingly, exosomes released from HUVECs treated with chemotherapeutic agents, epirubicin and PTX, were shown to induce antitumor responses during breast cancer chemotherapy. ${ }^{100}$ Using miRNA profiling, Bovy et al. identified specific miR-503, which exhibited downregulated levels in exosomes released from these endothelial cells. The exosome- 
incorporated miR-503 impaired breast cancer cells growth and decreased their invasive capacities.

In another study, adeno-associated viruses were encapsulated in EVs (e.g., vexosomes) that displayed viral capsid proteins. Notably, these vexosomes were efficient for the delivery of genetic cargo into recipient cells. ${ }^{101}$ Aside of RNA, the incorporation of exogenous DNA into EVs by electroporation was also reported. ${ }^{102,103}$ Interestingly, the loading efficiency and capacity of DNA in EVs was dependent on DNA size-linear DNA molecules less than $1000 \mathrm{bp}$ in length were more efficiently transferred into EVs compared with larger linear DNAs and plasmid DNAs. ${ }^{100}$ DNA-loaded EVs accomplished transfer of DNA into recipient cells in vitro. Overall, these reports indicate that exosomes may function as exceptional gene delivery vectors that are safe, efficient, organ/cell-specific, and nonimmunogenic.

Exosomes are also known to be nature's way of delivering different proteins. ${ }^{12}$ Consequently, loading of exosomes released from autologous macrophages with a therapeutic protein, catalase, ex vivo was shown to produce potent anti-inflammatory and neuroprotective effects in murine models of Parkinson's disease (PD). ${ }^{104}$ The optimal loading procedure included permeabilization of exosomal membranes with saponin in the presence of catalase. Notably, the loaded catalase was efficiently preserved against proteases degradation by proteases. A considerable amount of exosomes was detected in PD mouse brain following intranasal administration. This resulted in significant neuroprotective effects in in vitro and in vivo models of PD. It was hypothesized that the encapsulation of catalase into exosomes may preserve catalase enzymatic activity, prolong the blood circulation time, reduce immunogenicity, and improve its interaction with epithelial cells, thus improving drug transport and therapeutic effects in PD.

The genetic modification of donor cells may also be used for targeting exosomes to the disease site. As an example, targeting of exosomes to the brain was achieved by engineering the parental DCs to express lysosomal-associated membrane protein 2 (Lamp2b), fused to the neuron-specific peptide derived from rabies virus glycoprotein (RVG). ${ }^{94}$ Systemically administered RVG-targeted exosomes delivered glyceraldehyde 3-phosphate dehydrogenase (GAPDH) siRNA specifically to neurons, microglia, and oligodendrocytes in the brain, resulting in specific gene knockdown. The therapeutic potential of exosome-mediated siRNA delivery was demonstrated by the strong mRNA $(60 \%)$ and protein $(62 \%)$ knockdown of BACE1, a therapeutic target in $\mathrm{AD}$, in wild-type mice. ${ }^{94}$ Similar approach was applied when HEK 293T cell-derived exosomes expressing RVGLamp2b fusion protein were utilized to deliver opioid receptor $\mu$ (MOR) siRNA into the brain to treat morphine addiction. ${ }^{105}$ These exosomes were able to cross efficiently the BBB and transfer MOR siRNA into the mouse brain. The delivered siRNA inhibited morphine relapse via the downregulation of MOR expression levels, indicating a potential for the use of exosomes to treat drug addiction as well as other diseases of the central nervous system. Next, exosomes released from macrophages genetically modified to express antioxidant, catalase, or glial cells-derived neurotrophic factor were suggested for the treatment of PD. ${ }^{106,107}$ Mechanistic studies revealed that exosomes secreted from pretransfected macrophages contained the encoded therapeutic protein, as well as its genetic material (DNA and mRNA) and NF-kb, a transcription factor involved in the encoded gene expression. ${ }^{106}$ Drug-loaded exosomes were able to efficiently transfer their contents to contiguous neurons resulting in de novo protein synthesis in target cells. ${ }^{101}$ The transfected brain tissues showed monthlong expression of the encoded protein and prolonged attenuation of neuroinflammation (over 40 days) in mice with neuroinflammation.

This suggests that exosome-based formulations have a potential to be a versatile strategy to treat various disorders. Nevertheless, significant efforts are required and complex regulatory hurdles must be overcome in order to develop these therapies for clinical use.

\section{LIMITATIONS OF EXOSOME-BASED PRODUCTS}

Using exosomes as drug delivery vehicles takes advantage of their natural carriage and extraordinary ability to interact with target cells. It offers several benefits over common drug administration regimens; however, there are number of limitations and challenges that need to be addressed. One of the major challenges in developing exosome-based formulations is whether the sufficient number of exosomes can be generated. ${ }^{108}$ Indeed, the exosome yield per cell will impact the final production cost as well as clinical applications. In this respect, the choice of parental cells is critical. For example, MSCs are known to produce large amounts of exosomes, suggesting that these cells may be efficient for exosome production in a clinically applicable scale. ${ }^{109}$ Next, extended culturing of donor cells may considerably increase exosomal production. For example, culturing DCs for 
extended time period ${ }^{28}$ or at low $\mathrm{pH}^{110}$ can increase the exosomal production up to 10 -fold. In another study, the breakdown of parental cells (monocytes/ macrophages) loaded with anticancer agents, and isolation of exosome-like nanoparticles allowed a 100fold higher production yield of the drug carriers. ${ }^{80}$ Finally, specifically designed bioreactors that resemble bioreactors for tissue engineering ${ }^{111}$ can be utilized for exosomes scale-up. Importantly, different batches of exosomes can be pulled together, lyophilized, and stored until their use in patients. ${ }^{104}$

As an alternative approach, MSCs may be harvested from bone marrow, propagated in culture to obtain specific cell types, or even subtypes, and then exosomes may be loaded with a therapeutic agent. Although this approach would require a more invasive procedure, a significant amount of as well as storage of well-characterized exosomal carriers would be possible. ${ }^{112}$ Furthermore, large-scale production of therapeutically efficacious exosomes can be achieved through the immortalization of donor cells; for example, MSCs can be transfected by lentivirus carrying a MYC gene as reported in Ref 113. MYC is a regulator gene that codes for a transcription factor that plays a role in cell cycle progression. The transfection allows for obtaining immortalized cells but does not alter the fundamental characteristics of these MSCs. ${ }^{113}$ In this case, a library of various types of exosomal carriers for different drug formulations could be developed in the future and stored in stock for emergency situations. Finally, exosomes may be isolated from other sources (bovine milk and crashed grapes), purified, loaded with a drug, and used for oral or intranasal administration.

Next, efficient loading of exosomes without significant changes in the structure and content of exosomal membranes is also a difficult task, as exosomes consist of relatively tight and highly structured lipid bilayers. It should be taken into consideration that the disruption of the exosomes integrity during loading procedures might alter their immune-privileged status and, therefore, make them visible for the MPS. Furthermore, exosomal carriers may lose their own biological activity upon loading with a drug that may diminish their ability to target disease tissues and produce potent therapeutic effect. For example, we demonstrated that biologically active exosomes released by regenerative M2-subtype macrophages can produce a potent synergistic neuroprotective effect in mouse models of PD. ${ }^{104}$ Moreover, exosomes loaded with PTX were shown to target metastases in a model of murine LLC pulmonary metastases and efficiently eliminate cancer cells. ${ }^{86}$ It was hypothesized that macrophage-released exosomes are likely to have specific proteins on their surface, which might allow for their preferential accumulation in cancer cells. Therefore, preservation of lipid and protein content of exosomal carriers may be crucial for their therapeutic efficacy.

\section{REGULATORY POINTS FOR EXOSOME-BASED FORMULATIONS}

Before bringing exosomes to clinic, it is important for manufacturers to follow the appropriate FDA guidelines when producing exosome-based therapeutics. Exosome-based therapies most likely fall under the purview of The Office of Cellular, Tissue, and Gene Therapies (OCTGT) within the FDA Center for Biologics Evaluation and Research (CBER). The OCTGT regulates cellular therapies, gene therapies, therapeutic cancer vaccines, xenotransplants, combination products, and certain devices related to cell and gene therapy. Exosomes themselves compose a fairly heterogeneous population in terms of their biochemical composition, the source (different cell lines or patient samples) often dictates exosome phenotype ${ }^{114}$; this has critical implications for the use of exosomes in the clinic. It should also be noted that the absolute separation and definition of various EVs (including exosomes) based on their size or biogenesis has yet to be established beyond doubt, and there is currently no consensus on markers that distinguish the origin of these vesicles once they have left the cell. ${ }^{27}$

In order to manufacture exosomes, cell populations must be maintained for exosome harvest. Because of the diversity in donor and tissue source, certain information regarding the cell population must be provided in order to ensure safety to the patient. The type(s) of cells to be used should be classified as autologous, allogeneic, or xenogeneic in origin, and the tissue source or other relevant information should also be provided. For donors, any relevant characteristics of the donor(s) should be specified, including age and sex. If a mixture of cells or cell products from multiple donors is used, careful attention must be payed to possible cell interactions that may result in immune responses or other changes that might alter the performance of the cells. One must also include exclusion criteria for donors, focusing on the presence or likelihood of infection by HIV, hepatitis, etc. For autologous cells, one may refer to 62 CFR 9721 for additional guidance on adventitious agent testing and labeling. Cells should be handled, propagated, and subjected to laboratory procedures under conditions to minimize 
contamination, and documentation should be provided. Structural and biological information about the final exosome product should also be provided to show that an appropriate and biologically active form is present.

The manufacturing of exosomal formulations is the key in fulfilling the industrial development of exosomal drug formulations; purity, sterility, potency, and identity of the manufacturing lots are all crucial points to achieve in order to bring the drug to clinic and must be tested. The overall scheme for testing of exosome-based formulations is outlined in Figure 2. The OCTGT should offer a flexible approach to such testing due to the complex nature of exosome-based products, similar to the flexibility in testing allowed for cell-based therapies.

The reproducibility and consistency of the product lots may be difficult to achieve as the starting material may come from many different donors and thus possesses inherent variability. ${ }^{115}$ For example, Le Pecq ${ }^{116}$ evaluated the variability of the manufacturing process for over 100 separate exosomal lots from blood cells of healthy volunteers. The analysis of the data showed that the main source of variability was related to the heterogeneity of the human population, and not to the manufacturing process. Therefore, if the exosomal drug formulation to be administered to the patient is to be autologous, this may reduce the difficulty in achieving reproducible and consistent lots of exosomes.
The loading of drug into exosomes also represents a challenge in maintaining consistent and reproducible lots, depending on the method of drug loading and type of drug loaded into exosomes. If loading nucleic acids, there may be lot-to-lot variability in the purity and sequence of nucleic acids loaded, the transfection efficiency may possess variability due to impurities in the transfection reagent, and there may also be variability in the bioassays used to characterize drug loading. When loading small-molecule drugs, lot-to-lot variability of the drug may be a concern, as well as variability in the assays used to determine drug loading. Therefore, more rigorous characterization of exosome-based formulations using a combination of methods may be required.

Quality control of the lots is of paramount importance in order to ensure safety and reproducibility. Harvested exosomes should be assessed for impurities and consistency, and any reagents used to isolate or load exosomes with drug should undergo a qualification program in order to ensure that they are of the appropriate grade and quality for product consistency. The manufacturing should be monitored by in-process testing. Thus, the preparation of GMPgrade exosomes from the ascites fluid of ovarian cancer patients and the methods used for the molecular and immunological characterization of these exosomes preceding their use in clinical trials were described by Navabi et al. ${ }^{117}$ Quality assessment of the exosomes produced with this method has

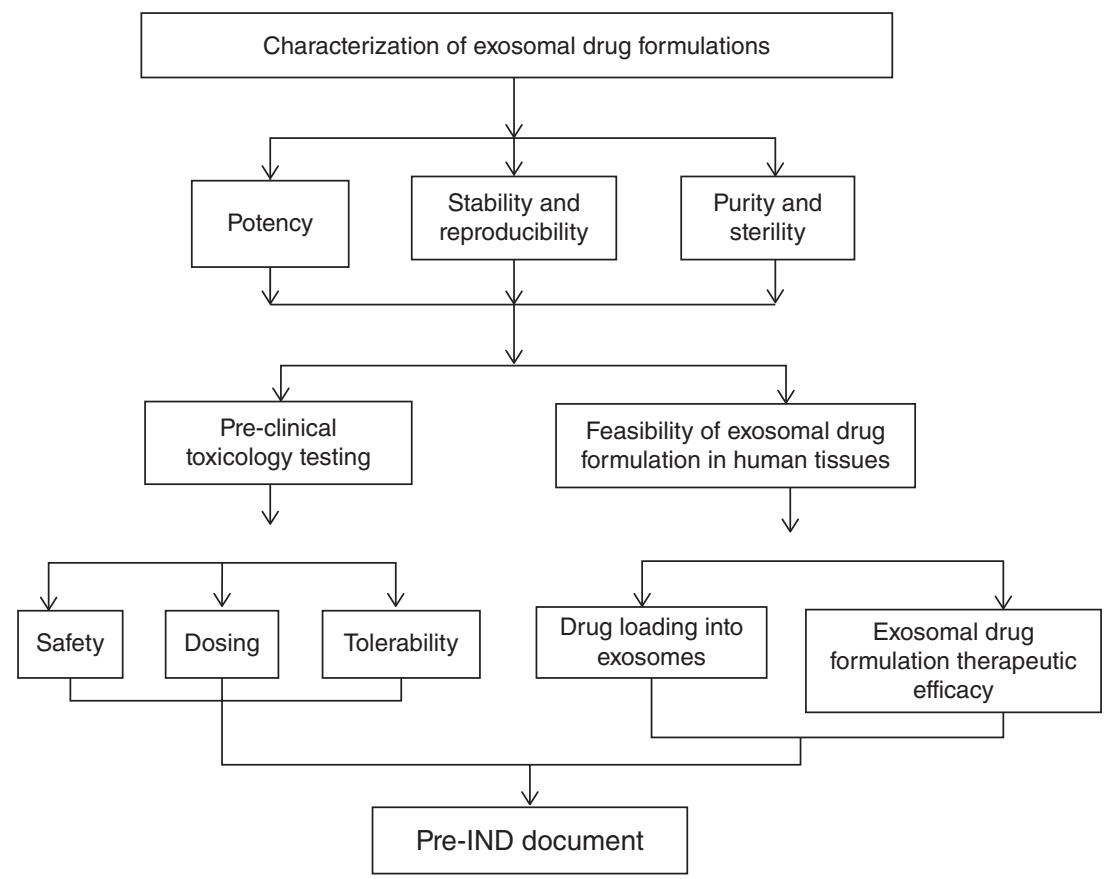

FIGURE 2 | The overall scheme of testing of exosome-based drug formulations in preclinical investigations. 
demonstrated the consistent expression of MHC Class I, CD81, and HSP70 molecules using Western blotting. Because there is no way to sterilize the exosomal product postprocessing, aseptic technique should be utilized throughout the manufacturing process to maintain sterility; the use of closed manufacturing systems is encouraged. Testing for microbial contamination is also imperative due to the biological nature of the exosome product, which requires extensive culture and manipulation.

The potency of exosomal drug formulations must also be measured. According to FDA standards, 'potency' is defined as the products' 'specific ability or capacity...to effect a given result." ${ }^{118}$ The FDA allows a 'matrix approach' to potency testing for cell-based therapies, which exosomes may technically fall under, looking at multiple characteristics and cumulatively assessing them as a measure of potency. Surrogate biomarkers (e.g., exosomal protein marker expression) that correlate with biological activity can be used to assess potency for exosome-based therapies, which do not have any added drug. Those with an added drug (e.g., PTX or curcumin) should also test the concentration of drug in the final product as well as activity of the final exosome product to assess potency. There is currently no consensus on exosome definitions and a lack of understanding regarding the mechanisms of action of various exosome-based therapies; thus, it may be difficult to assess the composition and bioactivity of the final exosome-based therapeutic product. The OCTGT recommends testing for characteristics that will help predicting the safety and efficacy of the final product.

Identity testing is required in order to distinguish one product from another produced at the same facility; however, this poses a challenge for cell therapy and exosomal products because identity testing may be unable to distinguish patient-specific lots from one another. Tracking, labeling, and segregation systems must be implemented in order to avoid mixing up patient-specific products. The FDA may require a hold on the exosomal product in order to complete identity and safety testing. This may also be challenging for cell therapy products as the hold time may affect their viability. However, exosome-based products have been shown to be stable for extended periods of time and are not as affected by the hold time as cell therapy products. Significantly, exosomes can be concentrated, lyophilized, and reconstituted in water solutions, as this study demonstrated. ${ }^{104}$ This will allow scalability, standardization, and consistency of manufacturing different lots of exosomal drug formulations, when a considerable amount and long-turn storage of exosomes might be required. It may still be wise to perform in-process testing during critical junctures of the manufacturing process in order to minimize the time it takes for the exosomal product to be safely administered to the patient as well as to collect valuable information about the manufacturing process.

Toxicological in vivo evaluations are also required to complete the safety examinations. Although a patient may receive autologous exosomes, ex vivo manipulations may cause alterations in the surface protein composition of the exosomes or their morphology or result in aggregation and other changes from the initial harvested naïve exosomes. These alterations may induce immune response or cause toxicity; thus, a thorough evaluation of their cytotoxicity, genotoxicity, and oxidative damage should be assessed during preclinical studies. Furthermore, exosomes themselves may induce an unwanted immune response in the patient. To avoid this effect, studies should be performed during clinical trials to assess the immunogenicity of the exosome-based therapy; antibody development should be assessed both directly after administration and at least 28 days afterward. Then, one should determine, if the results are linked to any adverse events, pharmacokinetics, or pharmacodynamics of the exosome-based therapeutics.

Overall, there is currently a high degree of variability in terms of exosome sources, manufacturing processes, and in vitro and in vivo product characterization, which poses a significant challenge to the clinical translation of exosome-based therapies. An established consensus or an FDA guidance regarding these facets of exosome production would go far in bringing exosome-based therapies to the clinic.

\section{CONCLUSION}

Exosome-based formulations are the next generation drug delivery mechanism that combines nanoparticle size with noncytotoxic effects, a high drug-carrying capacity, and a low immunogenic profile. Significant technological, functional, and safety features of this therapeutics are still to be addressed. Thus, deficiencies in our knowledge of molecular mechanisms of exosomes formation and a lack of methods to interfere with the packaging of cargo hamper identification of their physiological relevance in vivo. However, they promise an unparalleled efficacy in the treatment of many life-threatening conditions, including those lacking effective pharmacotherapy. 


\section{FURTHER READING}

Aikawa E, Gardiner C, Hutcheson JD, Ochiya T, Osteikoetxea X, Pegtel M, Piper M, Quesenberry P, Schiffelers RM, Szabo TG, et al. International Society for Extracellular Vesicles: Second Annual Meeting, 17-20 April 2013, Boston, MA (ISEV 2013). J Extracell Vesicles 2013, 2:23070.

\section{REFERENCES}

1. Peer D, Karp JM, Hong S, Farokhzad OC, Margalit R, Langer R. Nanocarriers as an emerging platform for cancer therapy. Nat Nanotechnol 2007, 2:751-760.

2. Davis ME, Zuckerman JE, Choi CH, Seligson D, Tolcher A, Alabi CA, Yen Y, Heidel JD, Ribas A. Evidence of RNAi in humans from systemically administered siRNA via targeted nanoparticles. Nature 2010, 464:1067-1070.

3. Peng Q, Zhang S, Yang Q, Zhang T, Wei XQ, Jiang L, Zhang CL, Chen QM, Zhang ZR, Lin YF. Preformed albumin corona, a protective coating for nanoparticles based drug delivery system. Biomaterials 2013, 34:8521-8530.

4. Beckman JS, Minor RL Jr, White CW, Repine JE, Rosen GM, Freeman BA. Superoxide dismutase and catalase conjugated to polyethylene glycol increases endothelial enzyme activity and oxidant resistance. J Biol Chem 1988, 263:6884-6892.

5. Yoshida K, Burton GF, McKinney JS, Young H, Ellis EF. Brain and tissue distribution of polyethylene glycol-conjugated superoxide dismutase in rats. Stroke 1992, 23:865-869.

6. Veronese FM, Caliceti P, Schiavon O, Sergi M. Polyethylene glycol-superoxide dismutase, a conjugate in search of exploitation. Adv Drug Deliv Rev 2002, 54:587-606.

7. Dams ET, Laverman P, Oyen WJ, Storm G, Scherphof GL, van Der Meer JW, Corstens FH, Boerman OC. Accelerated blood clearance and altered biodistribution of repeated injections of sterically stabilized liposomes. J Pharmacol Exp Ther 2000, 292:1071-1079.

8. Ishida T, Maeda R, Ichihara M, Irimura K, Kiwada H. Accelerated clearance of PEGylated liposomes in rats after repeated injections. I Control Release 2003, 88:35-42.

9. Ishida T, Kashima S, Kiwada H. The contribution of phagocytic activity of liver macrophages to the accelerated blood clearance (ABC) phenomenon of PEGylated liposomes in rats. J Control Release 2008, 126:162-165.

10. Pidaparti M, Bostrom B. Comparison of allergic reactions to pegasparaginase given intravenously versus intramuscularly. Pediatr Blood Cancer 2012, 59:436-439.

11. Andaloussi SEL, Mager I, Breakefield XO, Wood MJ. Extracellular vesicles: biology and emerging therapeutic opportunities. Nat Rev Drug Discov 2013, 12:347-357.

12. Raposo G, Stoorvogel W. Extracellular vesicles: exosomes, microvesicles, and friends. J Cell Biol 2013, 200:373-383.

13. Marcus ME, Leonard JN. FedExosomes: engineering therapeutic biological nanoparticles that truly deliver. Pharmaceuticals (Basel) 2013, 6:659-680.

14. Thery C, Amigorena S, Raposo G, Clayton A. Isolation and characterization of exosomes from cell culture supernatants and biological fluids. Curr Protoc Cell Biol 2006, Chapter 3:Unit 3.22.

15. Bhatnagar S, Shinagawa K, Castellino FJ, Schorey JS. Exosomes released from macrophages infected with intracellular pathogens stimulate a proinflammatory response in vitro and in vivo. Blood 2007, 110:3234-3244.

16. Clayton A, Turkes A, Navabi H, Mason MD, Tabi Z. Induction of heat shock proteins in B-cell exosomes. $J$ Cell Sci 2005, 118:3631-3638.

17. Nolte-'t Hoen EN, Buschow SI, Anderton SM, Stoorvogel W, Wauben MH. Activated T cells recruit exosomes secreted by dendritic cells via LFA-1. Blood 2009, 113:1977-1981.

18. Lai RC, Yeo RW, Lim SK. Mesenchymal stem cell exosomes. Semin Cell Dev Biol 2015, 40:82-88.

19. Song J, Chen X, Wang M, Xing Y, Zheng Z, Hu S. Cardiac endothelial cell-derived exosomes induce specific regulatory B cells. Sci Rep 2014, 4:7583.

20. Skogberg G, Lundberg V, Berglund M, Gudmundsdottir J, Telemo E, Lindgren S, Ekwall O. Human thymic epithelial primary cells produce exosomes carrying tissue-restricted antigens. Immunol Cell Biol 2015, 93:724-734.

21. Benito-Martin A, Di Giannatale A, Ceder S, Peinado H. The new deal: a potential role for secreted vesicles in innate immunity and tumor progression. Front Immunol 2015, 6:66. 
22. Muller L, Hong C-S, Stolz DB, Watkins SC, Whiteside TL. Isolation of biologically-active exosomes from human plasma. J Immunol Methods 2014, 411:55-65.

23. Taylor DD, Shah S. Methods of isolating extracellular vesicles impact down-stream analyses of their cargoes. Methods 2015, 87:3-10.

24. Taylor DD, Zacharias W, Gercel-Taylor C. Exosome isolation for proteomic analyses and RNA profiling. Methods Mol Biol 2011, 728:235-246.

25. Wang Z, Wu H-j, Fine D, Schmulen J, Hu Y, Godin B, Zhang JXJ, Liu X. Ciliated micropillars for the microfluidic-based isolation of nanoscale lipid vesicles. Lab Chip 2013, 13:2879-2882.

26. Liga A, Vliegenthart ADB, Oosthuyzen W, Dear JW, Kersaudy-Kerhoas M. Exosome isolation: a microfluidic road-map. Lab Chip 2015, 15:2388-2394.

27. Witwer KW, Buzás EI, Bemis LT, Bora A, Lässer C, Lötvall J, Nolte-'t Hoen EN, Piper MG, Sivaraman S, Skog J, et al. Standardization of sample collection, isolation and analysis methods in extracellular vesicle research. J Extracell Vesicles 2013, 27:2. doi:10.3402/ jev.v3402i3400.20360.

28. Lamparski HG, Metha-Damani A, Yao JY, Patel S, Hsu DH, Ruegg C, Le Pecq JB. Production and characterization of clinical grade exosomes derived from dendritic cells. J Immunol Methods 2002, 270:211-226.

29. Perez-Gonzalez R, Gauthier SA, Kumar A, Levy E. The exosome secretory pathway transports amyloid precursor protein carboxyl-terminal fragments from the cell into the brain extracellular space. J Biol Chem 2012, 287:43108-43115.

30. van der Pol E, Coumans FA, Grootemaat AE, Gardiner C, Sargent IL, Harrison P, Sturk A, van Leeuwen TG, Nieuwland R. Particle size distribution of exosomes and microvesicles determined by transmission electron microscopy, flow cytometry, nanoparticle tracking analysis, and resistive pulse sensing. J Thromb Haemost 2014, 12:1182-1192.

31. Lotvall J, Hill AF, Hochberg F, Buzas EI, Di Vizio D, Gardiner C, Gho YS, Kurochkin IV, Mathivanan S, Quesenberry P, et al. Minimal experimental requirements for definition of extracellular vesicles and their functions: a position statement from the International Society for Extracellular Vesicles. J Extracell Vesicles 2014, 3:26913.

32. Aalberts $M$, van Dissel-Emiliani FM, van Adrichem NP, van Wijnen M, Wauben MH, Stout TA, Stoorvogel W. Identification of distinct populations of prostasomes that differentially express prostate stem cell antigen, annexin A1, and GLIPR2 in humans. Biol Reprod 2012, 86:82.

33. Gyorgy B, Szabo TG, Pasztoi M, Pal Z, Misjak P, Aradi B, Laszlo V, Pallinger E, Pap E, Kittel A, et al. Membrane vesicles, current state-of-the-art: emerging role of extracellular vesicles. Cell Mol Life Sci 2011, 68:2667-2688.

34. Biancone L, Bruno S, Deregibus MC, Tetta C, Camussi G. Therapeutic potential of mesenchymal stem cell-derived microvesicles. Nephrol Dial Transplant 2012, 27:3037-3042.

35. Lai RC, Arslan F, Lee MM, Sze NS, Choo A, Chen TS, Salto-Tellez M, Timmers L, Lee CN, El Oakley RM, et al. Exosome secreted by MSC reduces myocardial ischemia/reperfusion injury. Stem Cell Res 2010, 4:214-222.

36. Arslan F, Lai RC, Smeets MB, Akeroyd L, Choo A, Aguor EN, Timmers L, van Rijen HV, Doevendans PA, Pasterkamp G, et al. Mesenchymal stem cell-derived exosomes increase ATP levels, decrease oxidative stress and activate PI3K/Akt pathway to enhance myocardial viability and prevent adverse remodeling after myocardial ischemia/reperfusion injury. Stem Cell Res 2013, 10:301-312.

37. Lee C, Mitsialis SA, Aslam M, Vitali SH, Vergadi E, Konstantinou G, Sdrimas K, Fernandez-Gonzalez A, Kourembanas S. Exosomes mediate the cytoprotective action of mesenchymal stromal cells on hypoxiainduced pulmonary hypertension. Circulation 2012, 126:2601-2611.

38. Katsuda T, Tsuchiya R, Kosaka N, Yoshioka Y, Takagaki K, Oki K, Takeshita F, Sakai Y, Kuroda M, Ochiya T. Human adipose tissue-derived mesenchymal stem cells secrete functional neprilysin-bound exosomes. Sci Rep 2013, 3:1197.

39. Xin H, Li Y, Chopp M. Exosomes/miRNAs as mediating cell-based therapy of stroke. Front Cell Neurosci 2014, 8:377.

40. Ilmer M, Vykoukal J, Recio Boiles A, Coleman M, Alt E. Two sides of the same coin: stem cells in cancer and regenerative medicine. FASEB J 2014, 28:2748-2761.

41. Emanueli C, Shearn AI, Angelini GD, Sahoo S. Exosomes and exosomal miRNAs in cardiovascular protection and repair. Vascul Pharmacol 2015, 71:24-30.

42. Huang L, Ma W, Ma Y, Feng D, Chen H, Cai B. Exosomes in mesenchymal stem cells, a new therapeutic strategy for cardiovascular diseases? Int J Biol Sci 2015, 11:238-245.

43. Deregibus MC, Cantaluppi V, Calogero R, Lo Iacono M, Tetta C, Biancone L, Bruno S, Bussolati B, Camussi G. Endothelial progenitor cell derived microvesicles activate an angiogenic program in endothelial cells by a horizontal transfer of mRNA. Blood 2007, 110:2440-2448.

44. Jeyaseelan K, Lim KY, Armugam A. MicroRNA expression in the blood and brain of rats subjected to transient focal ischemia by middle cerebral artery occlusion. Stroke 2008, 39:959-966.

45. Lusardi TA, Murphy SJ, Phillips JI, Chen Y, Davis CM, Young JM, Thompson SJ, Saugstad JA. 
MicroRNA responses to focal cerebral ischemia in male and female mouse brain. Front Mol Neurosci 2014, 7:11.

46. Liu FJ, Lim KY, Kaur P, Sepramaniam S, Armugam A, Wong PT, Jeyaseelan K. microRNAs involved in regulating spontaneous recovery in embolic stroke model. PLoS One 2013, 8:e66393.

47. Herrera MB, Fonsato V, Gatti S, Deregibus MC, Sordi A, Cantarella D, Calogero R, Bussolati B, Tetta C, Camussi G. Human liver stem cell-derived microvesicles accelerate hepatic regeneration in hepatectomized rats. J Cell Mol Med 2010, 14:1605-1618.

48. Tan CY, Lai RC, Wong W, Dan YY, Lim SK, Ho HK. Mesenchymal stem cell-derived exosomes promote hepatic regeneration in drug-induced liver injury models. Stem Cell Res Ther 2014, 5:76.

49. Hergenreider E, Heydt S, Treguer K, Boettger T, Horrevoets AJ, Zeiher AM, Scheffer MP, Frangakis AS, Yin X, Mayr M, et al. Atheroprotective communication between endothelial cells and smooth muscle cells through miRNAs. Nat Cell Biol 2012, 14:249-256.

50. Yellon DM, Davidson SM. Exosomes: nanoparticles involved in cardioprotection? Circ Res 2014, 114:325-332.

51. Sahoo S, Losordo DW. Exosomes and cardiac repair after myocardial infarction. Circ Res 2014, 114:333-344.

52. Ibrahim AG, Cheng K, Marban E. Exosomes as critical agents of cardiac regeneration triggered by cell therapy. Stem Cell Rep 2014, 2:606-619.

53. Aminzadeh MA, Tseliou E, Sun B, Cheng K, Malliaras K, Makkar RR, Marban E. Therapeutic efficacy of cardiosphere-derived cells in a transgenic mouse model of non-ischaemic dilated cardiomyopathy. Eur Heart J 2015, 36:751-762.

54. Ding Y, Wang J, Stierhof YD, Robinson DG, Jiang L. Unconventional protein secretion. Trends Plant Sci 2012, 17:606-615.

55. Regente M, Pinedo M, Elizalde M, de la Canal L. Apoplastic exosome-like vesicles: a new way of protein secretion in plants? Plant Signal Behav 2012, 7:544-546.

56. Ju S, Mu J, Dokland T, Zhuang X, Wang Q, Jiang H, Xiang X, Deng ZB, Wang B, Zhang L, et al. Grape exosome-like nanoparticles induce intestinal stem cells and protect mice from DSS-induced colitis. Mol Ther 2013, 21:1345-1357.

57. Robbins PD, Morelli AE. Regulation of immune responses by extracellular vesicles. Nat Rev Immunol 2014, 14:195-208.

58. Morse MA, Garst J, Osada T, Khan S, Hobeika A, Clay TM, Valente N, Shreeniwas R, Sutton MA, Delcayre A, et al. A phase I study of dexosome immunotherapy in patients with advanced non-small cell lung cancer. J Transl Med 2005, 3:9.

59. Escudier B, Dorval T, Chaput N, Andre F, Caby MP, Novault S, Flament C, Leboulaire C, Borg C, Amigorena $S$, et al. Vaccination of metastatic melanoma patients with autologous dendritic cell (DC) derived-exosomes: results of the first phase I clinical trial. J Transl Med 2005, 3:10.

60. Schorey JS, Cheng Y, Singh PP, Smith VL. Exosomes and other extracellular vesicles in host-pathogen interactions. EMBO Rep 2015, 16:24-43.

61. Zitvogel L, Regnault A, Lozier A, Wolfers J, Flament C, Tenza D, Ricciardi-Castagnoli P, Raposo G, Amigorena S. Eradication of established murine tumors using a novel cell-free vaccine: dendritic cell-derived exosomes. Nat Med 1998, 4:594-600.

62. Romagnoli GG, Zelante BB, Toniolo PA, Migliori IK, Barbuto JA. Dendritic cell-derived exosomes may be a tool for cancer immunotherapy by converting tumor cells into immunogenic targets. Front Immunol 2014, 5:692.

63. Qazi KR, Gehrmann U, Domange Jordo E, Karlsson MC, Gabrielsson S. Antigen-loaded exosomes alone induce Th1-type memory through a B-celldependent mechanism. Blood 2009, 113:2673-2683.

64. Kaur S, Singh SP, Elkahloun AG, Wu W, AbuAsab MS, Roberts DD. CD47-dependent immunomodulatory and angiogenic activities of extracellular vesicles produced by T cells. Matrix Biol 2014, 37:49-59.

65. van der Grein SG, Nolte-'t Hoen EN. "Small talk" in the innate immune system via RNA-containing extracellular vesicles. Front Immunol 2014, 5:542.

66. Aucher A, Rudnicka D, Davis DM. MicroRNAs transfer from human macrophages to hepato-carcinoma cells and inhibit proliferation. J Immunol 2013, 191:6250-6260.

67. Bruno S, Collino F, Deregibus MC, Grange C, Tetta C, Camussi G. Microvesicles derived from human bone marrow mesenchymal stem cells inhibit tumor growth. Stem Cells Dev 2013, 22:758-771.

68. Di Bonito P, Ridolfi B, Columba-Cabezas S, Giovannelli A, Chiozzini C, Manfredi F, Anticoli S, Arenaccio C, Federico M. HPV-E7 delivered by engineered exosomes elicits a protective CD8(+) T cellmediated immune response. Viruses 2015, 7:1079-1099.

69. Colino J, Snapper CM. Dendritic cell-derived exosomes express a Streptococcus pneumoniae capsular polysaccharide type 14 cross-reactive antigen that induces protective immunoglobulin responses against pneumococcal infection in mice. Infect Immun 2007, 75:220-230.

70. Schnitzer JK, Berzel S, Fajardo-Moser M, Remer KA, Moll H. Fragments of antigen-loaded dendritic cells (DC) and DC-derived exosomes induce protective immunity against Leishmania major. Vaccine 2010, 28:5785-5793. 
71. Admyre C, Johansson SM, Qazi KR, Filen JJ, Lahesmaa R, Norman M, Neve EP, Scheynius A, Gabrielsson S. Exosomes with immune modulatory features are present in human breast milk. J Immunol 2007, 179:1969-1978.

72. Zonneveld MI, Brisson AR, van Herwijnen MJ, Tan S, van de Lest CH, Redegeld FA, Garssen J, Wauben MH, Nolte-'t Hoen EN. Recovery of extracellular vesicles from human breast milk is influenced by sample collection and vesicle isolation procedures. $J$ Extracell Vesicles 2014, 3:1-12.

73. Kim SH, Bianco N, Menon R, Lechman ER, Shufesky WJ, Morelli AE, Robbins PD. Exosomes derived from genetically modified DC expressing FasL are anti-inflammatory and immunosuppressive. Mol Ther 2006, 13:289-300.

74. Peche H, Renaudin K, Beriou G, Merieau E, Amigorena S, Cuturi MC. Induction of tolerance by exosomes and short-term immunosuppression in a fully MHC-mismatched rat cardiac allograft model. Am J Transplant 2006, 6:1541-1550.

75. Urbanelli L, Buratta S, Sagini K, Ferrara G, Lanni M, Emiliani C. Exosome-based strategies for diagnosis and therapy. Recent Pat CNS Drug Discov 2015, 10:10-27.

76. De Toro J, Herschlik L, Waldner C, Mongini C. Emerging roles of exosomes in normal and pathological conditions: new insights for diagnosis and therapeutic applications. Front Immunol 2015, 6:203.

77. Lasser C. Exosomes in diagnostic and therapeutic applications: biomarker, vaccine and RNA interference delivery vehicle. Expert Opin Biol Ther 2015, 15:103-117.

78. Zhuang X, Xiang X, Grizzle W, Sun D, Zhang S, Axtell RC, Ju S, Mu J, Zhang L, Steinman L, et al. Treatment of brain inflammatory diseases by delivering exosome encapsulated anti-inflammatory drugs from the nasal region to the brain. Mol Ther 2011, 19:1769-1779.

79. Sun D, Zhuang X, Xiang X, Liu Y, Zhang S, Liu C, Barnes S, Grizzle W, Miller D, Zhang HG. A novel nanoparticle drug delivery system: the anti-inflammatory activity of curcumin is enhanced when encapsulated in exosomes. Mol Ther 2010, 18:1606-1614.

80. Jang SC, Kim OY, Yoon CM, Choi DS, Roh TY, Park J, Nilsson J, Lotvall J, Kim YK, Gho YS. Bioinspired exosome-mimetic nanovesicles for targeted delivery of chemotherapeutics to malignant tumors. ACS Nano 2013, 7:7698-7710.

81. Tian Y, Li S, Song J, Ji T, Zhu M, Anderson GJ, Wei J, Nie G. A doxorubicin delivery platform using engineered natural membrane vesicle exosomes for targeted tumor therapy. Biomaterials 2014, 35:2383-2390.

82. Rani S, Ryan AE, Griffin MD, Ritter T. Mesenchymal stem cell-derived extracellular vesicles: toward cell-free therapeutic applications. Mol Ther 2015, 23:812-823.
83. Yang T, Martin P, Fogarty B, Brown A, Schurman K, Phipps R, Yin VP, Lockman P, Bai S. Exosome delivered anticancer drugs across the blood-brain barrier for brain cancer therapy in Danio rerio. Pharm Res 2015, 32:2003-2014.

84. Pascucci L, Cocce V, Bonomi A, Ami D, Ceccarelli P, Ciusani E, Vigano L, Locatelli A, Sisto F, Doglia SM, et al. Paclitaxel is incorporated by mesenchymal stromal cells and released in exosomes that inhibit in vitro tumor growth: a new approach for drug delivery. $J$ Control Release 2014, 192:262-270.

85. Lee J, Kim J, Jeong M, Lee H, Goh U, Kim H, Kim B, Park JH. Liposome-based engineering of cells to package hydrophobic compounds in membrane vesicles for tumor penetration. Nano Lett 2015, 15:2938-2944.

86. Kim MS, Haney MJ, Zhao Y, Mahajan V, Deygen I, Klyachko NL, Inskoe E, Piroyan A, Sokolsky M, Okolie O, et al. Development of exosome-encapsulated paclitaxel to overcome MDR in cancer cells. Nanomedicine 2015. doi:10.1016/j.nano.2015.10.012. [Epub ahead of print].

87. Wahlgren J, De LKT, Brisslert M, Vaziri Sani F, Telemo E, Sunnerhagen P, Valadi H. Plasma exosomes can deliver exogenous short interfering RNA to monocytes and lymphocytes. Nucleic Acids Res 2012, 40:e130.

88. Shtam TA, Kovalev RA, Varfolomeeva EY, Makarov EM, Kil YV, Filatov MV. Exosomes are natural carriers of exogenous siRNA to human cells in vitro. Cell Commun Signal 2013, 11:88.

89. Pan Q, Ramakrishnaiah V, Henry S, Fouraschen S, de Ruiter PE, Kwekkeboom J, Tilanus HW, Janssen HL, van der Laan LJ. Hepatic cell-to-cell transmission of small silencing RNA can extend the therapeutic reach of RNA interference (RNAi). Gut 2012, 61:1330-1339.

90. Liu Y, Zhao L, Li D, Yin Y, Zhang CY, Li J, Zhang Y. Microvesicle-delivery miR-150 promotes tumorigenesis by up-regulating VEGF, and the neutralization of miR-150 attenuate tumor development. Protein Cell 2013, 4:932-941.

91. El Andaloussi S, Lakhal S, Mager I, Wood MJ. Exosomes for targeted siRNA delivery across biological barriers. Adv Drug Deliv Rev 2013, 65:391-397.

92. Lai CP, Breakefield XO. Role of exosomes/microvesicles in the nervous system and use in emerging therapies. Front Physiol 2012, 3:228.

93. Lai RC, Yeo RW, Tan KH, Lim SK. Exosomes for drug delivery-a novel application for the mesenchymal stem cell. Biotechnol Adv 2013, 31:543-551.

94. Alvarez-Erviti L, Seow Y, Yin H, Betts C, Lakhal S, Wood MJ. Delivery of siRNA to the mouse brain by systemic injection of targeted exosomes. Nat Biotechnol 2011, 29:341-345.

95. Thery C, Ostrowski M, Segura E. Membrane vesicles as conveyors of immune responses. Nat Rev Immunol 2009, 9:581-593. 
96. Waldenstrom A, Genneback N, Hellman U, Ronquist G. Cardiomyocyte microvesicles contain DNA/RNA and convey biological messages to target cells. PLoS One 2012, 7:e34653.

97. Lee Y, El Andaloussi S, Wood MJ. Exosomes and microvesicles: extracellular vesicles for genetic information transfer and gene therapy. Hum Mol Genet 2012, 21:R125-R134.

98. Ohno S, Takanashi M, Sudo K, Ueda S, Ishikawa A, Matsuyama N, Fujita K, Mizutani T, Ohgi T, Ochiya T, et al. Systemically injected exosomes targeted to EGFR deliver antitumor microRNA to breast cancer cells. Mol Ther 2013, 21:185-191.

99. O’Brien K, Lowry MC, Corcoran C, Martinez VG, Daly M, Rani S, Gallagher WM, Radomski MW, MacLeod RA, O'Driscoll L. miR-134 in extracellular vesicles reduces triple-negative breast cancer aggression and increases drug sensitivity. Oncotarget 2015, 6:32774-32789.

100. Bovy N, Blomme B, Freres P, Dederen S, Nivelles O, Lion M, Carnet O, Martial JA, Noel A, Thiry M, et al. Endothelial exosomes contribute to the antitumor response during breast cancer neoadjuvant chemotherapy via microRNA transfer. Oncotarget 2015, 6:10253-10266.

101. Maguire CA, Balaj L, Sivaraman S, Crommentuijn MH, Ericsson M, MinchevaNilsson L, Baranov V, Gianni D, Tannous BA, SenaEsteves M, et al. Microvesicle-associated AAV vector as a novel gene delivery system. Mol Ther 2012, 20:960-971.

102. Kanada M, Bachmann MH, Hardy JW, Frimannson DO, Bronsart L, Wang A, Sylvester MD, Schmidt TL, Kaspar RL, Butte MJ, et al. Differential fates of biomolecules delivered to target cells via extracellular vesicles. Proc Natl Acad Sci USA 2015, 112:E1433-E1442.

103. Lamichhane TN, Raiker RS, Jay SM. Exogenous DNA loading into extracellular vesicles via electroporation is size-dependent and enables limited gene delivery. Mol Pharm 2015, 12:3650-3657.

104. Haney MJ, Klyachko NL, Zhao Y, Gupta R, Plotnikova EG, He Z, Patel T, Piroyan A, Sokolsky M, Kabanov AV, et al. Exosomes as drug delivery vehicles for Parkinson's disease therapy. $J$ Control Release 2015, 207:18-30.

105. Liu Y, Li D, Liu Z, Zhou Y, Chu D, Li X, Jiang X, Hou D, Chen X, Chen Y, et al. Targeted exosomemediated delivery of opioid receptor $\mathrm{Mu}$ siRNA for the treatment of morphine relapse. Sci Rep 2015, $5: 17543$

106. Haney MJ, Zhao Y, Harrison EB, Mahajan V, Ahmed S, He Z, Suresh P, Hingtgen SD, Klyachko NL, Mosley RL, et al. Specific transfection of inflamed brain by macrophages: a new therapeutic strategy for neurodegenerative diseases. PLoS One 2013, 8:e61852.

107. Zhao Y, Haney MJ, Gupta R, Bohnsack JP, He Z, Kabanov AV, Batrakova EV. GDNF-transfected macrophages produce potent neuroprotective effects in Parkinson's disease mouse model. PLoS One 2014, 9:e106867.

108. Nordin JZ, Lee Y, Vader P, Mager I, Johansson HJ, Heusermann W, Wiklander OP, Hallbrink M, Seow Y, Bultema JJ, et al. Ultrafiltration with size-exclusion liquid chromatography for high yield isolation of extracellular vesicles preserving intact biophysical and functional properties. Nanomedicine 2015, 11:879-883.

109. Yeo RW, Lai RC, Zhang B, Tan SS, Yin Y, Teh BJ, Lim SK. Mesenchymal stem cell: an efficient mass producer of exosomes for drug delivery. Adv Drug Deliv Rev 2013, 65:336-341.

110. Ban JJ, Lee M, Im W, Kim M. Low pH increases the yield of exosome isolation. Biochem Biophys Res Commun 2015, 461:76-79.

111. Portner R, Nagel-Heyer S, Goepfert C, Adamietz P, Meenen NM. Bioreactor design for tissue engineering. J Biosci Bioeng 2005, 100:235-245.

112. Muller FJ, Snyder EY, Loring JF. Gene therapy: can neural stem cells deliver? Nat Rev Neurosci 2006, $7: 75-84$.

113. Chen TS, Arslan F, Yin Y, Tan SS, Lai RC, Choo AB, Padmanabhan J, Lee CN, de Kleijn DP, Lim SK. Enabling a robust scalable manufacturing process for therapeutic exosomes through oncogenic immortalization of human ESC-derived MSCs. J Transl Med 2011, 9:47.

114. Chevillet JR, Kang Q, Ruf IK, Briggs HA, Vojtech LN, Hughes SM, Cheng HH, Arroyo JD, Meredith EK, Gallichotte EN, et al. Quantitative and stoichiometric analysis of the microRNA content of exosomes. Proc Natl Acad Sci USA 2014, 111:14888-14893.

115. Patel S, Mehta-Damani A, Shu H, Le Pecq JB. An analysis of variability in the manufacturing of dexosomes: implications for development of an autologous therapy. Biotechnol Bioeng 2005, 92:238-249.

116. Le Pecq JB. Dexosomes as a therapeutic cancer vaccine: from bench to bedside. Blood Cells Mol Dis 2005, 35:129-135.

117. Navabi H, Croston D, Hobot J, Clayton A, Zitvogel L, Jasani B, Bailey-Wood R, Wilson K, Tabi Z, Mason MD, et al. Preparation of human ovarian cancer ascites-derived exosomes for a clinical trial. Blood Cells Mol Dis 2005, 35:149-152.

118. U.S. Department of Health and Human Services Food and Drug Administration. Guidance for industry: providing clinical evidence of effectiveness for human drug and biological products, 1998. 